\title{
SIGN REGULARITY OF MACLAURIN COEFFICIENTS OF FUNCTIONS IN THE LAGUERRE-PÓLYA CLASS
}

\author{
DIMITAR K. DIMITROV AND WILLIAN D. OLIVEIRA
}

\begin{abstract}
We prove that the signs of the Maclaurin coefficients of a wide class of entire functions that belong to the Laguerre-Pólya class posses a regular behaviour.
\end{abstract}

\section{INTRODUCTION}

A real entire function $\varphi$ is in the Laguerre-Pólya class, written $\varphi \in \mathcal{L P}$, if

$$
\varphi(z)=c z^{m} e^{a z^{2}+b z} \prod_{k=1}^{\omega}\left(1-z / x_{k}\right) e^{\lambda z / x_{k}}, \quad 0 \leq \omega \leq \infty,
$$

for some nonnegative integer $m, c, a, b \in \mathbb{R}, a \leq 0, \lambda \in\{0,1\}$ and $x_{k} \in \mathbb{R} \backslash\{0\}$ such that $\sum_{k}\left|x_{k}\right|^{-\lambda-1}<\infty$. When $\omega=0$ the product on the right-hand side of (1.1) is defined to be identically one. Otherwise the terms of the product are arranged according to the increasing order of $\left|x_{k}\right|$.

Observe that $\varphi \in \mathcal{L P}$ if and only if $\varphi(z)=\exp \left(a z^{2}\right) \phi(z)$, where $a \leq 0$ and $\phi$ is a real entire function with real zeros of genus at most one.

If $a=\lambda=0$ and $b \alpha_{k} \leq 0$ for every $k \in \mathbb{N}$ in the representation of $\varphi(z)$, the function $\varphi(z)$ is said to belong to the Laguerre Polya Class of type $I$, denoted by $\varphi \in \mathcal{L P} I$. In other words, $\varphi \in \mathcal{L P} I$ if and only if either $\varphi(z)$ or $\varphi(-z)$ can be represented in the form

$$
\varphi(z)=c z^{m} e^{b z} \prod_{k=1}^{\omega}\left(1-z / x_{k}\right),
$$

where $c \in \mathbb{R}, m \in \mathbb{N} \cup\{0\}, b \leq 0, x_{k}>0$ and $\sum_{k} x_{k}^{-1}<\infty$.

The functions in $\mathcal{L P}$ and only these are uniform limits on the compact sets of the complex plane (locally uniform limits) of hyperbolic polynomials, that is, real polynomials with only real zeros. Similarly, the functions in $\mathcal{L P I}$ are the locally uniform limits of hyperbolic polynomials with zeros of the same sign.

The class $\mathcal{L P}$ of functions was studied first by Laguerre [4] and later then by Pólya, Jensen, Schur, Obrechkoff (see [5, 6] and the references therein) and other celebrated mathematicians in the beginning of the twentieth century because of the attempts to settle the Riemann hypothesis. The connection between the latter and the LaguerrePólya Class is straightforward and we refer to [2, 3] for the details.

The properties of the functions in the Laguerre-Pólya class are tightly connected with the notion of multiplier sequence. A real sequence $\left\{\gamma_{n}\right\}_{n=0}^{\infty}$ is called a multiplier

2010 Mathematics Subject Classification. 30D10, 30D15.

Key words and phrases. Laguerre-Pólya class of entire functions, Maclaurin coefficients, multiplier sequence, sign regularity.

Research supported by by the Brazilian foundations CNPq under Grant 307183/2013-0 and FAPESP under Grant 2013/14881-9. 
sequence if for any hyperbolic polynomial

$$
p(z)=a_{0}+a_{1} z+\cdots+a_{m} z^{m}
$$

with zeros of the same sign, the polynomial

$$
q(z)=a_{0} \gamma_{0}+a_{1} \gamma_{1} z+\cdots+a_{n} \gamma_{m} z^{m}
$$

is a hyperbolic polynomial. Similarly, the sequence $\left\{\gamma_{n}\right\}_{n=0}^{\infty}$ is said to be a multiplier sequence of type $I$ if for any hyperbolic polynomial $p$ of the form (1.2), the polynomial $q$ given by (1.3) is a hyperbolic polynomial too. Pólya and Schur [7] proved that the sequence $\left\{\gamma_{n}\right\}_{n=0}^{\infty}$ is a multiplier sequence if and only if the series

$$
\sum_{n=0}^{\infty} \frac{\gamma_{n}}{n !} z^{n}
$$

represents an entire function in $\mathcal{L P}$ class. In particular the sequence $\left\{\gamma_{n}\right\}_{n=0}^{\infty}$ is a multiplier sequence of type $I$ if and only if the latter series represents an entire function in $\mathcal{L P} I$ class.

Therefore, given a real entire function with Maclaurin expansion

$$
\varphi(z)=\sum_{n=0}^{\infty} a_{n} z^{n}
$$

it is of interest to provide necessary and/or sufficient conditions in terms of the behaviour of the sequence $\left\{a_{n}\right\}$ in order that $\varphi$ belongs to $\mathcal{L P}$. In the present note we establish some results of this nature. We are interested if the signs of the coefficients $a_{n}$ exhibit some regular patterns. For this purpose it is convenient to divide $\mathcal{L P}$ into three subclasses. The first one is simply $\mathcal{L P}$. The second class, denoted by $\mathcal{L P}^{a}$ consists of function in $\mathcal{L P}$ for which the constant $a$ in the exponent in the representation (1.1) is nonzero. The third class consists of entire function in $\mathcal{L P}$ for which $a=0$ but do not belong to $\mathcal{L P} I$ and is denoted by $\mathcal{L} \mathcal{P}^{0}$.

It is straightforward that the Maclaurin coefficients of a nonpolynomial function $\varphi \in \mathcal{L P I}$ are either all of the same sign or their signs alternate. In fact, Pólya and Schur [7] proved that a real entire function in the Laguerre-Pólya class which exhibits this sign regularity of its coefficients is necessarily in $\mathcal{L P} I$.

However, Laguerre 4 provided a beautiful example of a parametric family of entire functions in the Laguere-Pólya class whose coefficients may exhibit signs form a rather irregular sequence for certain choices of the parameters. He proved that

$$
\varphi(z)=\sum_{n=0}^{\infty} \frac{\cos (\vartheta+n \theta)}{n !} z^{n}
$$

is in $\mathcal{L P}$. In fact, it is straightforward to observe that

$$
\varphi(z)=e^{z \cos \theta} \cos (\vartheta+z \sin \theta) .
$$

We emphasise that $a=0$ and $\varphi \notin \mathcal{L P} I$ because its zeros are not of the same sign. Therefore $\varphi \in \mathcal{L P}^{0}$. Varying the choices of $\vartheta$ and $\theta$ we observe a great variety of possibilities for the distribution of the signs in the sequence $\cos (\vartheta+n \theta)$, especially when $\theta / 2 \pi$ is an irrational number. Laguerre's example shows that one can hardly expect regularity in the distribution of the signs of the Maclaurin coefficients of a function in $\mathcal{L} \mathcal{P}^{0}$, similar to the one in $\mathcal{L P} I$.

The situation changes dramatically when one considers the class $\mathcal{L} \mathcal{P}^{a}$. The presence of the factor $\exp \left(a z^{2}\right), a<0$, seems to put order in the distribution of the signs of $a_{n}$. 
Let us consider three illustrative examples. The signs of the first Maclaurin coefficients of $\exp \left(-z^{2}\right) \exp (z), \exp \left(-z^{2}\right)\left(1 / \Gamma(z)\right.$ and $\exp \left(-z^{2}\right) \cos \sqrt{z}$ are

$\{1,1,-1,-1,1,1,1,-1,-1,1,1,-1,-1,1,1,-1,-1,1,1,-1,-1,-1,1,1,-1,-1,1,1\}$, $\{0,1,1,-1,-1,1,1,-1,-1,1,1,-1,1,1,-1,-1,1,1,-1,-1,1,1,-1,-1,1,1,-1,-1\}$, $\{1,-1,-1,1,1,-1,-1,1,1,-1,-1,1,1,-1,-1,1,1,-1,-1,1,1,-1,-1,1,1,-1,-1\}$, respectively. Observe that the common pattern $\{1,1,-1,-1\}$ appears frequently. A detailed analysis of these and a vast number of other function in $\mathcal{L} \mathcal{P}^{a}$ led us to observe that this phenomenon occurs infinitely many times. This pattern can be formalized stating that the inequalities $a_{n-1} a_{n+1}<0$ hold asymptotically. These observations made us pose the following:

Question A. Let $\varphi(z)=\sum_{n=0}^{\infty} a_{n} z^{n}$ be a real entire function with only real zeros. Is it true that $\varphi \in \mathcal{L} \mathcal{P}^{a}$ if and only if $0<\limsup n\left|a_{n}\right|^{2 / n}<\infty$ and there exists $n_{0} \in \mathbb{N}$, such that $a_{n-1} a_{n+1} \leq 0$ for all $n>n_{0}$ ?

We have substituted the strict inequalities $a_{n-1} a_{n+1}<0$ by $a_{n-1} a_{n+1} \leq 0$ in order to cover the possibility of occurence of zeros in the sequence $a_{n}$ which happens, for instance, when the entire function is either even or odd. We establish a partial results towards an affirmative answer to Question A proving that the inequalities $a_{n-1} a_{n+1} \leq 0$, together with adequate asymptotic rate of $a_{n}$ guarantees that a real entire function with real zeros indeed belongs to $\mathcal{L P}^{a}$. Formally, one of our main results reads as:

Theorem 1. Suppose that the real function

$$
\varphi(z)=\sum_{n=0}^{\infty} a_{n} z^{n}
$$

possesses only real zeros. If there exist $n_{0} \in \mathbb{N}$, such that $a_{n-1} a_{n+1} \leq 0$ for all $n>n_{0}$ and $0<\lim \sup _{n \rightarrow \infty} n\left|a_{n}\right|^{2 / n}<\infty$, then $\varphi \in \mathcal{L P}^{a}$.

We state a relevant result concerning the necessity statement of the question too.

Theorem 2. If

$$
\varphi(z)=\sum_{n=0}^{\infty} a_{n} z^{n} \in \mathcal{L} \mathcal{P}^{a}
$$

can be represented in the form

$$
\varphi(z)=\exp \left(a z^{2}\right) P(z)
$$

where $a<0$ and $P$ is a hyperbolic polynomial, then $0<\lim \sup _{n \rightarrow \infty} n\left|a_{n}\right|^{2 / n}<\infty$ and there exists $n_{0} \in \mathbb{N}$, such that $a_{n-1} a_{n+1} \leq 0$ for every $n>n_{0}$.

However, the pattern of the signs of the coefficients of functions in $\mathcal{L P}^{a}$ we observed before stating the above question, admits exceptions. Hence the answer to Question $\mathrm{A}$ is negative. Moreover, exceptions occur rather frequently, for entire function in $\mathcal{L P}^{a}$ of any possible order, as seen in the following:

Theorem 3. For every $\rho$ with $0 \leq \rho \leq 2$, there is an entire function $\Psi(z)$ of order $\rho_{\Psi}=\rho$, of the form

$$
\psi(z)=\prod_{n=1}^{\infty}\left(1-\frac{z}{x_{n}}\right) \exp \left(\frac{\lambda z}{x_{n}}\right)
$$


such that, for every $a<0$, the function

$$
\varphi(z)=\exp \left(a z^{2}\right) \psi(z)=\sum_{n=0}^{\infty} a_{n} z^{n}
$$

is in $\mathcal{L P}^{a}$ and possesses the property that for every $n_{0} \in \mathbb{N}$, there is $n>n_{0}$, such that $a_{n-1} a_{n+1}>0$.

\section{Preliminaries}

Despite that most of the fact in this section are basic in the theory of entire functions and can be found in classical reading as 1, 5, we shall list them in the form we need in the proofs in order to make the reading relatively self-contained.

Given a sequence of nonzero complex numbers $\left\{z_{n}\right\}_{n \in \mathbb{N}}$, without accumulation points, we consider:

(i) the associated canonic product

$$
f(z)=\prod_{n=1}^{\infty} G\left(\frac{z}{z_{n}} ; p\right),
$$

where $p$ is the genus of the canonic product, that is, the smallest integer for which

$$
\sum_{n=1}^{\infty} \frac{1}{\left|z_{n}\right|^{p+1}}
$$

converges and

$$
G(u ; p)=(1-u) e^{u+u^{2} / 2+\cdots+u^{p} / p} ;
$$

(ii) the exponent of convergence $\lambda$ of the sequence as the infimum of all positive numbers $t$ such that

converges;

$$
\sum_{n=1}^{\infty} \frac{1}{\left|z_{n}\right|^{t}}
$$

(iii) the superior density $\Delta$ of the sequence defined by

$$
\Delta=\limsup _{r \rightarrow \infty} \frac{n(r)}{r^{\lambda}},
$$

where $n(r)=\#\left\{n:\left|z_{n}\right| \leq r\right\}$.

As it is well known, an entire function

$$
f(z)=\sum_{n=0}^{\infty} a_{n} z^{n}
$$

is of order $\rho_{f}$ if

$$
\limsup _{r \rightarrow \infty} \frac{\log \log M(r)}{\log r}=\rho_{f},
$$

where $M(r)=M_{f}(r)=\max _{|z|=r}|f(z)|$. If $\rho_{f}<\infty$, then $f$ is said to be of type $\sigma_{f}$ if

$$
\limsup _{r \rightarrow \infty} \frac{\log M(r)}{r^{\rho}}=\sigma_{f} .
$$


When $0<\rho_{f}<\infty$ the order and the type are determined in terms of the coefficients via

$$
\begin{gathered}
\rho_{f}=-\limsup _{n \rightarrow \infty} \frac{n \log n}{\log \left|a_{n}\right|}, \\
\sigma_{f}=\limsup _{n \rightarrow \infty} \frac{n \sqrt[n]{\left|a_{n}\right|^{\rho_{f}}}}{e \rho_{f}} .
\end{gathered}
$$

Borel's theorem claims that the order of a canonic product is equal to the exponent of convergence of its zeros.

The category $\kappa_{f}$ of the entire function $f$ is the pair of its order and type, that is $\kappa_{f}=\left(\rho_{f}, \sigma_{f}\right)$. The entire functions are partially ordered (denoted $\preceq$ ) according to alphabetic order of their categories. Precisely, $f$ and $g$ belong to the same category provided both their orders and types coincide. Otherwise, if $\rho_{f}>\rho_{g}$ then $f$ belongs to a higher category. Finally, if $\rho_{f}=\rho_{g}$ but $\sigma_{f}>\sigma_{g}$ then $f$ is of a higher category. The so-called Theorem of categories (see [5. Theorem 12, p. 23]) states if the entire functions $f$ and $g$ belong to distinct categories, then the product $f g$ belongs to the category of the factor with the higher category. In other words,

$$
\kappa_{f g}=\max \left\{\kappa_{f}, \kappa_{g}\right\} .
$$

Hadamard's theorem claims that every entire function $f$ of finite order $\rho_{f}$ can be represented in the form

$$
f(z)=z^{k} \exp (P(z)) \prod_{n=1}^{\infty} G\left(\frac{z}{z_{n}} ; p\right)
$$

where $k \in \mathbb{N} \cup\{0\}, P$ is an algebraic polynomial of degree not exceeding $\rho_{f}$, and $z_{n}$ are the zeros of $f$ distinct from the origin.

We state the classical theorem of Lindelöf in its complete form, as in [5, Theorem 15, p. 28].

Theorem A. (Lindelöf) Let $f$ be an entire function with Hadamard product (2.8), of finite order $\rho_{f}$, type $\sigma_{f}$, genus $p$ of its canonic product and superior density of its zeros $\Delta_{f}$. Then the following hold:

(i) If $\rho_{f}$ is not integer, then $\Delta_{f}$ and $\sigma_{f}$ are simultaneously equal to either 0 , a finite number, or $\infty$.

(ii) If $\rho_{f}$ is an integer and $\rho_{f}>p$, then $f$ is of type $\sigma_{f}=\left|\alpha_{\rho_{f}}\right|$, where $\alpha_{\rho_{f}}$ is the coefficient of $z^{\rho_{f}}$ in the expansion of the polynomial $P(z)$.

(iii) If $\rho_{f}=p$ and

$$
\delta_{f}(r)=\left|\alpha_{\rho_{f}}+\frac{1}{\rho_{f}} \sum_{\left|z_{n}\right|<r} z_{n}^{-\rho_{f}}\right|, \quad \bar{\delta}_{f}=\limsup _{r \rightarrow \infty} \delta_{f}(r), \quad \gamma_{f}=\max \left(\Delta_{f}, \bar{\delta}_{f}\right),
$$

then $\sigma_{f}$ and $\gamma_{f}$ are simultaneously equal to either 0 , a finite number, or $\infty$.

Finally, the classical Hermite-Biehler theorem states that all zeros of the algebraic polynomial

$$
r(z)=u(z)+i v(z),
$$

where $u(z)$ and $v(z)$ are polynomials with real coefficients, belong to one of the open semi-planes determined by the real axis, if and only if both $u$ and $v$ are hyperbolic polynomials and their zeros interlace. 


\section{Proofs}

We begin this section with a technical lemma which shows how to determine the order and the type of an entire function from the asymptotic behaviour of its Taylor coefficients. The result might be of independent interest and the idea of the proof goes back to [5, Theorem 2, p. 4].

Lemma 1. Let $\rho>0$ and $f(z)=\sum_{n=0}^{\infty} a_{n} z^{n}$ be an entire function, such that $0<$ $\limsup _{n \rightarrow \infty}\left(n \sqrt[n]{\left|a_{n}\right| \rho}\right)<\infty$. Then $\rho_{f}=\rho$ and $0<\sigma_{f}<\infty$.

Proof. The proof goes by reductio ad absurdum. First we assume that $\rho_{f}>\rho$. It follows from $\limsup _{n \rightarrow \infty}\left(n \sqrt[n]{\left|a_{n}\right|^{\rho}}\right)<\infty$ that there is $K>0$, such that

$$
\frac{n \sqrt[n]{\left|a_{n}\right|^{\rho}}}{\rho e}<K \text { for } n>n(K)
$$

or equivalently,

$$
\left|a_{n}\right|<\left(\frac{\rho e K}{n}\right)^{n / \rho} \text { for } n>n(K) .
$$

Let $|z|=r$, where $r>r(K)$ is large enough so that the inequality $N(r)=\left[2^{\rho} \rho e K r^{\rho}\right]>$ $n(K)$ holds. Here [.] stands for the integer part. Then we employ the estimate (3.9) to obtain

$$
\begin{aligned}
|f(z)| & \leq \sum_{n=0}^{N(r)}\left|a_{n}\right| r^{n}+\sum_{n=N(r)+1}^{\infty}\left|a_{n}\right| r^{n} \\
& \leq \sum_{n=0}^{N(r)}\left|a_{n}\right| r^{n}+2^{-N(r)} \\
& <(N(r)+1) \max _{n}\left\{a_{n} r^{n}\right\}+2^{-N(r)} .
\end{aligned}
$$

Hence,

$$
M_{f}(r)<\left(2^{\rho} \rho e K r^{\rho}+1\right) \max _{n}\left\{a_{n} r^{n}\right\}+1 \text { for } r>r(K) .
$$

Observe that $f$ is not a polynomial because we assumed that $\rho_{f}>\rho>0$. Then the Cauchy estimate yields that $M_{f}(r)$ increases faster than any power of $r$. The latter inequality implies that $\max _{n}\left\{a_{n} r^{n}\right\}$ increases faster than any power of $r$ too. This means that the index $n$, where the maximum is attained increases going to infinity as $r \rightarrow \infty$. Redefining $r(K)$, if necessary, so that the index $n$ where $\max _{n}\left\{a_{n} r^{n}\right\}$ occurs be such that $n>n(K)$, we obtain

$$
\max _{n}\left\{a_{n} r^{n}\right\} \leq\left(\frac{\rho e K}{n}\right)^{n / \rho} r^{n}, \quad r>r(K) .
$$

A straightforward analysis show that the maximum on the right-hand side of the latter is attained at $n=\rho K r^{\rho}$. Thus

$$
\max _{n}\left\{a_{n} r^{n}\right\} \leq e^{K r^{\rho}}, \quad r>r(K) .
$$

Then

$$
M_{f}(r)<\left(2^{\rho} \rho e K r^{\rho}+2\right) e^{K r^{\rho}} \text { for } r>r(K),
$$

and, increasing $r(K)$ if necessary,

$$
M_{f}(r)<e^{2 K r^{\rho}}, \quad r>r(K) .
$$


On the other hand, the definition of the order of an entire function and the assumption that $\rho_{f}>\rho$ show that we may chose $\epsilon_{K}>0$ in such a way that $\rho_{f}-\epsilon_{K}>\rho$ and $r^{\rho_{f}-\epsilon_{K}-\rho}>2 K$ for every $r>r\left(\epsilon_{K}\right)$ and

$$
M_{f}(r)>\exp \left(r^{\rho_{f}-\epsilon_{k}}\right)=\exp \left(r^{\rho_{f}-\epsilon_{K}-\rho^{\rho}} r^{\rho}>\exp \left(2 K r^{2}\right), \quad r>r\left(\epsilon_{K}\right) .\right.
$$

The latter contradicts (3.10).

Assume now that $\rho_{f}<\rho$. Then, given $\epsilon>0$, there exists $r(\epsilon)$ such that

$$
M_{f}(r) \leq \exp \left(\epsilon r^{\rho}\right), \quad r>r(\epsilon) .
$$

By the Cauchy estimate

$$
\left|a_{n}\right| \leq \frac{M_{f}(r)}{r^{n}} \leq \frac{\exp \left(\epsilon r^{\rho}\right)}{r^{n}}, \quad r>r(\epsilon) .
$$

The minimum of $r^{-n} \exp \left(\epsilon r^{\rho}\right)$, when $r>0$, is attained at $r=(n / \epsilon \rho)^{1 / \rho}$. Choose now $n(\epsilon)$ such that $(n / \epsilon \rho)^{1 / \rho}>r(\epsilon)$ for all $n>n(\epsilon)$, to obtain

$$
\left|a_{n}\right| \leq\left(\frac{\rho \epsilon e}{n}\right)^{n / \rho}, \quad n>n(\epsilon),
$$

which is equivalent to

$$
n \sqrt[n]{\left|a_{n}\right|^{\rho}}<\rho \epsilon e, \quad n>n(\epsilon)
$$

Since the above reasonings hold for any $\epsilon>0$, then

$$
\limsup _{n \rightarrow \infty}\left(n \sqrt[n]{\left|a_{n}\right|^{\rho}}\right)=0
$$

This a contradiction to the hypothesis that $\limsup _{n \rightarrow \infty}\left(n \sqrt[n]{\left|a_{n}\right|^{\rho}}\right)>0$.

Therefore, $\rho_{f}=\rho$. The fact that $0<\sigma_{f}<\infty$ follows from (2.6).

Proof. (of Theorem 1) By Lemma 11 $\rho_{\varphi}=2$ and $0<\sigma_{\varphi}<\infty$. Hadamard's factorisation theorem implies that $\varphi$ can be represented as

$$
\varphi(z)=c z^{k} \exp \left(a z^{2}+b z\right) \prod_{n=1}^{\infty} G\left(\frac{z}{x_{n}} ; p\right)
$$

where $k \in \mathbb{N} \cup\{0\}, a, b, c \in \mathbb{R}$, and $x_{n}$ are the zeros of $\varphi$ distinct from 0 . We shall prove that the series

$$
\sum_{n=1}^{\infty} \frac{1}{x_{n}^{2}}
$$

converges. Let $\rho_{\Pi}$ and $\sigma_{\Pi}$ be the order and the type of the canonic product in (3.11). Since $\rho_{\varphi}=2$, then (2.7) implies

$$
\kappa_{\Pi} \preceq\left(2, \sigma_{\varphi}\right) .
$$

Assume that the series (3.12) diverges. Then the exponent of convergence $\lambda_{\Pi}$ of the canonic product in (3.11) obeys $\lambda_{\Pi} \geq 2$. It follows from the above mentioned theorem of Borel and (3.13) that $\lambda_{\Pi}=\rho_{\Pi}=2$. Since (3.12) diverges, then we must have $p=2$. Hence $\rho_{\Pi}=p$ and, by item (iii) of Teorema

$$
\sigma_{\Pi}=\limsup _{r \rightarrow \infty}\left(\frac{1}{2} \sum_{\left|x_{n}\right|<r} \frac{1}{x_{n}^{2}}\right)=\infty .
$$

Therefore, $\kappa_{\Pi}=(2, \infty)$, so that, by (3.13),$\kappa_{\varphi}=(2, \infty)$. This contradicts $\sigma_{\varphi}<\infty$. Hence, the series (3.12) converges. 
The convergence of (3.12) implies that $p \leq 1$ and that (3.11) can be written in the form

$$
\varphi(z)=c z^{k} \exp \left(a z^{2}+b z\right) \prod_{n=1}^{\infty}\left(1-\frac{z}{x_{n}}\right) \exp \left(\frac{\lambda z}{x_{n}}\right),
$$

with $k \in \mathbb{N} \cup\{0\}, a, b, c \in \mathbb{R}$, where $x_{n} \in \mathbb{R}$ for every $n \in \mathbb{N}, \lambda \in\{0,1\}$ and $\sum\left(1 / x_{n}\right)^{\lambda+1}<\infty$. It remain to prove only that $a<0$ in (3.14). Assume the contrary, that $a \geq 0$.

However, there is $n_{0}$, such that $a_{n-1} a_{n+1} \leq 0$ for all $n>n_{0}$. Then we can write $\varphi(z)=\sum_{n=0}^{\infty} a_{n} z^{n}$ in the form

$$
\varphi(z)=P_{n_{0}}(z)+g(z)
$$

with a polynomial $P_{n_{0}}(z)$ of degree $n_{0}$ and $g$ an entire function of the form

$$
g(z)=\sum_{n=0}^{\infty} \operatorname{sgn}\left(b_{n}\right)\left|b_{n}\right| z^{n} \quad \text { with } \operatorname{sgn}\left(b_{n-1}\right) \operatorname{sgn}\left(b_{n+1}\right) \leq 0 \text { for all } n>0 .
$$

If $t>0$ then

$$
\begin{aligned}
|g(i t)| & =\left|\sum_{n=0}^{\infty} \operatorname{sgn}\left(b_{n}\right)\right| b_{n}\left|(i t)^{n}\right|=\left|\operatorname{sgn}\left(b_{0}\right) \sum_{n=0}^{\infty}\right| b_{2 n}\left|t^{2 n}+i \operatorname{sgn}\left(b_{1}\right) \sum_{n=0}^{\infty}\right| b_{2 n+1}\left|t^{2 n+1}\right| \\
& =\sqrt{\left(\sum_{n=0}^{\infty}\left|b_{2 n}\right| t^{2 n}\right)^{2}+\left(\sum_{n=0}^{\infty}\left|b_{2 n+1}\right| t^{2 n+1}\right)^{2}} \\
& \geq \frac{1}{\sqrt{2}} \sum_{n=0}^{\infty}\left|b_{n}\right| t^{n} \geq \frac{1}{\sqrt{2}} \max _{|z|=t}|g(z)|=\frac{1}{\sqrt{2}} M_{g}(t) .
\end{aligned}
$$

Since $\rho_{\varphi}=2>p$, then item (ii) of Theorem @yields $0<\sigma_{\varphi}=a$. Now (2.6) allows us to conclude that $\rho_{g}=2$ and $\sigma_{g}=a>0$. This implies that there is $t_{0}>0$, such that

$$
M_{g}(t)>\exp \left((a / 2) t^{2}\right) \text { and } \frac{\sqrt{2}\left|P_{n_{0}}(i t)\right|}{\exp \left((a / 2) t^{2}\right)}<\frac{1}{2} \text { for every } t>t_{0} .
$$

Therefore

$$
\begin{aligned}
|\varphi(i t)| & \geq|g(i t)|-\left|P_{n_{0}}(i t)\right| \geq \frac{1}{\sqrt{2}} M_{g}(t)-\left|P_{n_{0}}(i t)\right| \\
& \geq \frac{1}{\sqrt{2}} \exp \left((a / 2) t^{2}\right)\left(1-\frac{\sqrt{2}\left|P_{n_{0}}(i t)\right|}{\exp \left((a / 2) t^{2}\right)}\right) .
\end{aligned}
$$

Finally we conclude that

$$
|\varphi(i t)|>\frac{1}{2 \sqrt{2}} \exp \left((a / 2) t^{2}\right) \text { for every } t>t_{0} .
$$

The convergence of the series (3.12) shows that there are two possibilities: either $\rho_{\Pi}<2$ or $\rho_{\Pi}=2$ and, in the latter case, $\sigma_{\Pi}=0$ because of item (ii) of Theorem $\mathrm{A}$ In both cases, there is $t_{1}>0$, such that

$$
\left|\prod_{n=1}^{\infty}\left(1-\frac{i t}{a_{n}}\right) \exp \left(\frac{\lambda i t}{a_{n}}\right)\right|<\exp \left((a / 2) t^{2}\right) \text { and }|c| t^{k} \exp \left(-(a / 2) t^{2}\right)<1 \text { for } t>t_{1} \text {. }
$$


Using this inequality in (3.14) we obtain

$|\varphi(i t)|=|c| t^{k} \exp \left(-a t^{2}\right)\left|\prod_{n=1}^{\infty}\left(1-\frac{i t}{\alpha_{n}}\right) \exp \left(\frac{\lambda i t}{\alpha_{n}}\right)\right|<|c| t^{k} \exp \left(-(a / 2) t^{2}\right)<1, t>t_{1}$.

This contradicts (3.16). Therefore $a<0$ in (3.14) and $\varphi \in \mathcal{L P}^{a}$.

Corollary 1. Let $\varphi(z)=\sum_{n=0}^{\infty} a_{n} z^{n}$ be an even (odd) function with only real zeros. Then $\varphi \in \mathcal{L P}^{a}$ if and only if $0<\lim \sup \left(n \sqrt[n]{\left|a_{n}\right|^{2}}\right)<\infty$ and $a_{n-1} a_{n+1} \leq 0$ for every $n \in \mathbb{N}$.

Proof. If $0<\lim \sup \left(n \sqrt[n]{\left|a_{n}\right|^{2}}\right)<\infty$ and $a_{n-1} a_{n+1} \leq 0$ for every $n \in \mathbb{N}$, then, by Theorem 1 $\varphi \in \mathcal{L P}^{a}$.

Suppose that $\varphi \in \mathcal{L} \mathcal{P}^{a}$ is even (odd). It can be written in the form

$$
\varphi(z)=c z^{k} \exp \left(a z^{2}\right) \prod_{n=1}^{\infty}\left(1-\frac{z^{2}}{x_{n}^{2}}\right) .
$$

It suffices to apply Cauchy's multiplication formula to see that $a_{n-1} a_{n+1} \leq 0$ for all $n \in \mathbb{N}$. Since $\sum_{n=1}^{\infty}\left(1 / x_{n}^{2}\right)<\infty$, then item (ii) of Theorem $₫$ yields $\sigma_{\varphi}=-a$. Finally, (2.6) implies $0<\lim \sup \left(n \sqrt[n]{\left|a_{n}\right|^{2}}\right)<\infty$.

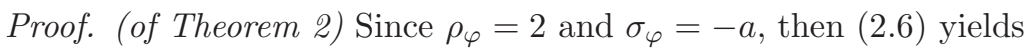

$$
0<\lim \sup \left(n \sqrt[n]{\left|a_{n}\right|^{2}}\right)<\infty .
$$

Let

$$
P(z)=\sum_{n=0}^{2 m} b_{n} z^{n}
$$

with the obvious convention that if $P$ is of odd degree, then $b_{2 m}=0$. Consider another polynomial,

$$
P_{1}(z)=a^{m} b_{0}+a^{m-1} b_{2} z+\cdots+b_{2 m} z(z-1) \ldots(z-m+1) .
$$

There exists $k_{0} \in \mathbb{N}$, such that $P_{1}(x)$ is strictly positive or strictly negative for $x>k_{0}$. Let $n=2 k+1$ be an odd number with $k>k_{0}$ and $k \geq m$. The Cauchy multiplication formula yields

$$
\begin{aligned}
a_{n-1} & =\sum_{j=0}^{m} \frac{a^{k-j}}{(k-j) !} b_{2 j}=\frac{a^{k-m}}{k !} \sum_{j=0}^{m} a^{m-j} b_{2 j} k(k-1) \ldots(k-j+1) \\
& =\frac{a^{k-m}}{k !} P_{1}(k), \\
a_{n+1} & =\sum_{j=0}^{m} \frac{a^{k+1-j}}{(k+1-j) !} b_{2 j}=\frac{a^{k+1-m}}{(k+1) !} \sum_{j=0}^{m} a^{m-j} b_{2 j}(k+1)(k) \ldots(k-j+2) \\
& =\frac{a^{k+1-m}}{(k+1) !} P_{1}(k+1) .
\end{aligned}
$$

Having in mind that $a<0$ and $P_{1}(k)$ and $P_{1}(k+1)$ have equal signs when $k>k_{0}$, we see that

$$
a_{n-1} a_{n+1}=\frac{a^{2(k-m)+1}}{k !(k+1) !} P_{1}(k) P_{1}(k+1) \leq 0,
$$


for all odd indices $n>2 k_{0}+1$. When $n$ is even, it suffices to apply an analogous argument but employing the polynomial

$$
P_{2}(x)=a^{m-1} b_{1}+a^{m-2} b_{3}(x-1)+\cdots+b_{2 m-1}(x-1)(x-2) \ldots(x-m+1)
$$

instead of $P_{1}$. Again, there is $n_{0}$, such that $a_{n-1} a_{n+1} \leq 0$ for all $n>n_{0}$.

Now we establish a lemma whose proof might be of independent interest because it is simpler than the proof of the corresponding general claim about functions in the Hermite-Biehler space.

Lemma 2. Let $\varphi(z)=\sum_{n=0}^{\infty} a_{n} z^{n}$ be an entire function with only real zeros, all of the same sign and $0 \leq \rho_{\varphi}<1$. Then all the zeros of both

$$
\begin{aligned}
& \varphi^{o}(z)=\sum_{n=0}^{\infty} a_{2 n+1} z^{2 n+1}, \\
& \varphi^{e}(z)=\sum_{n=0}^{\infty} a_{2 n} z^{2 n}
\end{aligned}
$$

are purely imaginary.

Proof. Since $0 \leq \rho_{\varphi}<1$, then the Hadamard product of $\varphi$ is

$$
\varphi(z)=\varphi(0) \prod_{n=1}^{\infty}\left(1-\frac{z}{x_{n}}\right) .
$$

Clearly, $\varphi$ is a local uniform limit of hyperbolic polynomials

$$
P_{m}(z)=\varphi(0) \prod_{n=1}^{m}\left(1-\frac{z}{x_{n}}\right)=\sum_{n=0}^{\infty} a_{2 n, m} z^{2 n}+\sum_{n=0}^{\infty} a_{2 n+1, m} z^{2 n+1},
$$

with $a_{n, m} \in \mathbb{R}$ and $a_{2 n, m}=a_{2 n+1, m}=0$, when $2 n>m$. Then obviously

$$
P_{m}(i z)=\varphi(0) \prod_{n=1}^{m}\left(1-\frac{i z}{x_{n}}\right)=\sum_{n=0}^{\infty}(-1)^{n} a_{2 n, m} z^{2 n}+i \sum_{n=0}^{\infty}(-1)^{n} a_{2 n+1, m} z^{2 n+1} .
$$

For each fixed $m \in \mathbb{N}$, the zeros of the polynomial $P_{m}(i z)$ are $-i x_{1}, \ldots,-i x_{m}$ and they posses imaginary parts of the same sign because the zeros $x_{n}$ of $\varphi$ are all of the same sign. By the Theorem of Hermite-Biehler, the zeros of both $\sum_{n=0}^{\infty}(-1)^{n} a_{2 n, m} z^{2 n}$ and $\sum_{n=0}^{\infty}(-1)^{n} a_{2 n+1, m} z^{2 n+1}$ are all real [6]. Therefore, the zeros of $\sum_{n=0}^{\infty} a_{2 n, m} z^{2 n}$ and $\sum_{n=0}^{\infty} a_{2 n+1, m} z^{2 n+1}$ are all purely imaginary. Since these polynomials converge locally uniformly to the functions $\varphi^{o}(z)$ and $\varphi^{e}(z)$, when $m \rightarrow \infty$, it follows from the Theorem of Hurwitz, that the zeros of $\varphi^{o}(z)$ e $\varphi^{e}(z)$ are purely imaginary too.

Proof. (of Theorem [3). First we shall prove that if $\psi$ is an entire function with the property that at least one of the functions

$$
\psi^{o}(z)=\sum_{n=0}^{\infty} b_{2 n+1} z^{2 n+1} \text { and } \psi^{e}(z)=\sum_{n=0}^{\infty} b_{2 n} z^{2 n}
$$

has an infinite number of purely imaginary zeros, then, for any $a<0$, the function

$$
\varphi(z)=\exp \left(a z^{2}\right) \psi(z)=\sum_{n=0}^{\infty} a_{n} z^{n}
$$


obeys the property that for every $n_{0} \in \mathbb{N}$, there exists $n>n_{0}$, such that $a_{n-1} a_{n+1}>0$. Indeed, for a given $a<0$, at least one of the functions

$$
\begin{gathered}
\varphi^{o}(z)=\sum_{n=0}^{\infty} a_{2 n+1} z^{2 n+1}=\exp \left(a z^{2}\right) \sum_{n=0}^{\infty} b_{2 n+1} z^{2 n+1}, \\
\varphi^{e}(z)=\sum_{n=0}^{\infty} a_{2 n} z^{2 n}=\exp \left(a z^{2}\right) \sum_{n=0}^{\infty} b_{2 n} z^{2 n}
\end{gathered}
$$

is not a polynomial and possesses infinitely many purely imaginary zeros. Suppose, without loss of generality, that this is $\varphi_{2}$, that is, $\varphi^{e}(z)=\sum_{n=0}^{\infty} a_{2 n} z^{2 n}$ is an entire function and it is not a polynomial. Assume that there exists $n_{0} \in \mathbb{N}$ such that $a_{n-1} a_{n+1} \leq 0$ for all $n>n_{0}$. Then we may represent it as

$$
\varphi^{e}(z)=P_{n_{0}}(z)+h(z),
$$

where $P_{n_{0}}(z)$ is a polynomial of degree $n_{0}$ and

$$
h(z)=\sum_{n=0}^{\infty} \operatorname{sgn}\left(c_{2 n}\right)\left|c_{2 n}\right| z^{2 n}
$$

is an entire function with $\operatorname{sgn}\left(c_{2 n}\right) \operatorname{sgn}\left(c_{2(n+1)}\right) \leq 0$ for every $n \geq 0$. Then for any $t \in \mathbb{R}$ we have

$$
\begin{aligned}
|h(i t)| & =\left|\sum_{n=0}^{\infty} \operatorname{sgn}\left(c_{2 n}\right)\right| c_{2 n}\left|(i t)^{n}\right|=\left.\left.\left|\operatorname{sgn}\left(c_{0}\right) \sum_{n=0}^{\infty}\right| c_{2 n}|| t\right|^{2 n}\left|=\sum_{n=0}^{\infty}\right| c_{2 n}|| t\right|^{2 n} \\
& \geq \max _{|z|=|t|}|h(z)|=M_{h}(|t|) .
\end{aligned}
$$

Let $\left\{i t_{m}\right\}$ be the sequence of the purely imaginary zeros of $\varphi^{e}$. Then (3.17) and (3.18) imply

$$
M_{h}\left(\left|t_{m}\right|\right) \leq\left|P_{n_{0}}\left(i t_{m}\right)\right|,
$$

for all $m \in \mathbb{N}$. The fact that $\varphi^{e}$ is an entire function shows that $\left|t_{m}\right| \rightarrow \infty$ as $m \rightarrow \infty$. Thus, there exists $m_{0}>0$, such that $\left|P_{n_{0}}\left(i t_{m}\right)\right|<\left|t_{m}\right|^{n_{0}+1}$, for all $m>m_{0}$. On the other hand, it follows from the Cauchy estimate that

$$
\left|c_{2 n}\right| \leq \frac{M_{h}\left(\left|t_{m}\right|\right)}{t_{m}^{2 n}} \leq \frac{\left|P_{n_{0}}\left(i t_{m}\right)\right|}{t_{m}^{2 n}} \leq \frac{\left|t_{m}\right|^{n_{0}+1}}{t_{m}^{2 n}}, \quad m>m_{0} .
$$

However, the latter implies that $c_{2 n}=0$ for all $n>\left(n_{0}+1\right) / 2$. Then, by (3.17), the function $\varphi_{2}$ is a polynomial, a contradiction.

Back to the proof of the theorem itself, suppose first that $\rho=0$. Consider a sequence $x_{n}$ of real positive numbers with exponent of convergence $\lambda=0$. We may consider, for instance, the sequence $x_{n}=e^{n}$. Then the canonic product

$$
\Pi_{1}(z)=\prod_{n=1}^{\infty}\left(1-\frac{z}{x_{n}}\right)
$$

is of order $\rho_{\Pi_{1}}=0$. By Lemma 2 the functions $\Pi_{1}^{o}(z)$ e $\Pi_{1}^{e}(z)$ have only purely imaginary zeros. Set $\psi(z)=\Pi_{1}(z)$. By the claim we have proved in the beginning the function $\psi$ is such that $\rho_{\psi}=\rho=0$ and, for each $a<0$, the function $\varphi(z)=$ $\exp \left(a z^{2}\right) \psi(z)=\sum_{n=0}^{\infty} a_{n} z^{n} \in \mathcal{L} \mathcal{P}^{a}$ possesses the property that for every $n_{0} \in \mathbb{N}$, there exists $n>n_{0}$, such that $a_{n-1} a_{n+1}>0$.

In the case $0<\rho \leq 2$, consider the sequence $y_{n}$ of real numbers, symmetric with respect to the origin, with exponent of convergence $\lambda=\rho$ and such that $\sum\left(1 /\left|y_{n}\right|^{2}\right)$ 
converges. We may consider, for instance, the sequence, $y_{2 n-1}=-(n \log (n+1))^{1 / \rho}$ and $y_{2 n}=(n \log (n+1))^{1 / \rho}$. Then the canonic product

$$
\Pi_{2}(z)=\prod_{n=1}^{\infty}\left(1-\frac{z}{y_{n}}\right) \exp \left(\frac{\lambda z}{y_{n}}\right),
$$

with $\lambda \in\{0,1\}$, represents an even entire function. Choose now $\psi(z)=\Pi_{2}(z) \Pi_{1}(z)$. Since $\psi^{o}(z)=\Pi_{2}(z) \Pi_{1}^{o}(z)$ and $\psi^{e}(z)=\Pi_{2}(z) \Pi_{1}^{e}(z)$, the real and the imaginary parts of $\psi$ possess an infinite number of purely imaginary zeros. Therefore $\psi(z)=\Pi_{2}(z) \Pi_{1}(z)$ is such that, for each $a<0$, the function $\varphi(z)=\exp \left(a z^{2}\right) \psi(z)=\sum_{n=0}^{\infty} a_{n} z^{n}$ obeys the property that for every $n_{0} \in \mathbb{N}$, there exists $n>n_{0}$, such that $a_{n-1} a_{n+1}>0$. Finally, observe that arranging the sequence $\left\{z_{n}\right\}$ of the zeros of $\Pi_{1}(z) \Pi_{2}(z)$ in an increasing order of their absolute values,

$$
\psi(z)=\prod_{n=1}^{\infty}\left(1-\frac{z}{z_{n}}\right) \exp \left(\frac{\lambda z}{z_{n}}\right),
$$

with $\lambda \in\{0,1\}$ and $\sum\left(1 /\left|z_{n}\right|^{\lambda+1}\right)$ convergent. Therefore, $\varphi(z)=\exp \left(a z^{2}\right) \psi(z) \in$ $\mathcal{L} \mathcal{P}^{a}$.

\section{REFERENCES}

[1] R. P. Boas, Entire Functions, Academic Press Inc, New York, 1954.

[2] G. Csordas and R. Varga, Necessary and sufficient conditions and the Riemann hypothesis, Adv. Appl. Math. 11 (1990), 328-357.

[3] D. K. Dimitrov and P. K. Rusev, Zeros of entire Fourier transforms, East J. Approx. 17 (2011), $1-110$.

[4] E. N. Laguerre, Oeuvres, Vol. I, Gauthier-Villan, Paris, 1898.

[5] B. Ya. Levin, Distribution of Zeros of Entire Functions, Amer. Math. Soc, 1980.

[6] N. Obrechkoff, Zeros of Polynomials, Marin Drinov Acad. Publ. House, Sofia, 2003.

[7] G. Pólya and I. Schur, Über zwei Arten von Faktorenfolgen in der Theorie der algebraischen Gleichungen, J. Reine Angew. 144 (1914), 89-113.

Departamento de Matemática Aplicada, iBilCE, Universidade Estadual Paulista, 15054000 SaÕ José do Rio Preto, SP, Brazil.

E-mail address: dimitrov@ibilce.unesp.br

Departamento de Matemática Aplicada, ibilce, Universidade Estadual Paulista, 15054000 Saõ José do Rio Preto, SP, Brazil.

E-mail address: wdoliveira@ibilce.unesp.br 